Cancer Metastasis Rev. 2014 September ; 33(0): 619-628. doi:10.1007/s10555-013-9480-2.

\title{
Bone-targeting agents in prostate cancer
}

\author{
Daniel L. Suzman, Sosipatros A. Boikos, and Michael A. Carducci \\ Prostate Cancer Research Program, Sidney Kimmel Comprehensive Cancer Center at Johns \\ Hopkins, 1650 Orleans Street, CRB1-1 M45, Baltimore, MD 21231-1000, USA
}

\begin{abstract}
Bone metastases are present in the vast majority of men with advanced prostate cancer, representing the main cause for morbidity and mortality. Recurrent or metastatic disease is managed initially with androgen deprivation but the majority of the patients eventually will progress to castration-resistant prostate cancer, with patients developing bone metastases in most of the cases. Survival and growth of the metastatic prostate cancer cells is dependent on a complex microenvironment (onco-niche) that includes the osteoblasts, the osteoclasts, the endothelium, and the stroma. This review summarizes agents that target the pathways involved in this complex interaction between prostate cancer and bone micro-environment and aim to transform lethal metastatic prostate cancer into a chronic disease.
\end{abstract}

\section{Keywords}

Bone-targeting agents; Prostate cancer; Bone metastases

\section{Introduction}

\begin{abstract}
Metastatic disease to the bone causes the majority of the morbidity and mortality associated with prostate cancer, leading to skeletal-related events (SREs) that include pathologic fractures, severe pain, and risk of spinal cord compromise. There remains a strong rationale to design agents that target the pathways involved in the development of bone metastases. Over the last few years, several novel agents have been approved by the US Food and Drug Administration (FDA) for use in advanced prostate cancer. Chemotherapy with docetaxel/ prednisone [1] was the first agent approved for the treatment of metastatic castrationresistant prostate cancer that improved survival. Since then, several agents, including the immunotherapeutic agent sipuleucel-T [2], cabazitaxel [3], radium-223 [4], and the androgen pathway-targeted agents, abiraterone [5] and enzalutamide [6], have been approved by the FDA, each agent demonstrating improved overall survival in large randomized phase 3 studies. As the biology and natural history of prostate cancer provide a strong rationale to target metastatic bone disease, this review will discuss several of these agents in the context of the bone microenvironment as well as agents targeting additional relevant pathways.
\end{abstract}

(C) Springer Science+Business Media New York 2014

carducci@jhmi.edu. 


\section{Prostate cancer in the bone microenvironment}

Stephen Paget proposed in 1889 that metastasis depends on the cross-talk between metastasizing cancer cells (the seeds) and the organ microenvironment (the soil) [7]. The bone microenvironment encompasses both osteoclasts and osteo-blasts, whose interaction of resorbing and reforming bone maintains skeletal integrity. In normal bone homeostasis, osteoclasts differentiate from hematopoetic stem cell precursors and adhere to bone, where subsequent resorption releases chemotactic cytokines that attract osteoblasts. Osteoblasts, derived from stromal stem cells, produce osteoid matrix and express both receptor activator of nuclear factor-kB ligand (RANKL) and osteoprotegerin. RANKL binds to the RANK transmembrane receptor on osteoclasts leading to osteoclast differentiation while osteoprotegerin functions as a decoy receptor, sequestering RANKL, helping to modulate the balance between destruction and production. Downstream of RANK in osteoclasts, activation of NF-kB leads to activation of osteoclast genes that lead to maturation, adherence to bone, and secretion of proteins involved in bone catabolism. These proteins include cathepsin $\mathrm{K}$, a protease that catabolizes bone collagen and promotes further osteoclast adhesion, and Src, a tyrosine kinase that is activated in osteoclasts after integrin binding to the bone matrix and which appears to promote osteoclast survival and formation of the "ruffled borders" and cell polarization needed for bone resorption $[8,9]$.

Osteoclasts and osteoblasts have been shown to interact with metastatic prostate cancer deposits to generate the "fertile soil" for prostate tumor cell homing. Along with endothelial cells, mesenchymal stem cells, adipocytes, and CXCL12-rich reticular cells, they create a local "onco-niche" that promotes prostate cancer bone metastasis development. Prostate cancer cells metastasize from the primary tumor to the circulation, where osteoblast-secreted cytokines such as SDF-1 (CXCL12) may function as a chemokine to the CXCR4 and CXCR7 receptors located on the prostate cancer cell surface, leading to adhesion molecule expression and homing to the bone, in effect co-opting the normal homing mechanism of hematopoetic stem cells [10]. This has led to an understanding of disseminated tumor cells (DTCs) acting as parasites, targeting the stem cell niche to invade and then proliferate to overt metastases [11].

Once within the bone microenvironment, a prostate tumor cell may then become dormant or proliferate into an overt metastatic mass with the release of cytokines and growth factors, including parathyroid hormone-related peptide, Wnt, vascular endothelial growth factor (VEGF), and bone morphogenic protein, that promote adhesion and proliferation to the bone. One factor in this branching point may be the specific location of bone, as for example, epiphyseal trabecular bone is a far more common site for metastasis than in the diaphysis [12]. While the precise mechanism for this tropism has not been definitively established, two pathways, annexin II and the growth-arrest specific 6 (GAS6) pathway may be involved. Annexin II, expressed on the surface of osteoblasts, regulates the adhesion and migration of prostate cancer cells to osteoblasts. It may further be a mediator of the induction of dormancy as well as drug resistance; blockade of this pathway decreased prostate cancer tumor burden in xenograft models [13]. Binding of GAS6 to its receptor, Axl, alters cellular functions including migration, invasion, proliferation, and survival. When prostate cancer cells bind to osteoblasts, displacing hematopoetic stem cells from the 
bone microenvironment, they upregulate Axl, leading to growth inhibition. A later shift to increased Tyro3 expression, an alternate GAS6 receptor, and decreased Axl expression results in increased growth and gross metastatic disease [14].

Once established within the bone microenvironment, tumor cells adhere and secrete paracrine factors at sites of increased bone turnover that stimulate osteoblasts and stromal cells to proliferate, differentiate, and produce growth factors, pathologically increasing RANKL levels. Excessive osteoclast activity leads to release of bone matrix factors, including endothelin-1 (ET-1), TGF- $\beta$ and TNF- $\alpha$, which promote growth and survival of tumor cells. Osteosclerotic lesions, despite increased osteoblast activity, also display increased osteoclast activity as measured by elevated $\mathrm{N}$-telopeptide levels. They progress via increased osteoblastogenesis and type-I collagen production with cytokines including TGF- $\beta$ and ET-1 leading to the formation of fragile woven bone, weakened structural integrity, and subsequent SREs $[15,16]$ (Fig. 1).

\section{Agents targeting bone homeostasis (osteoclast-osteoblast equilibrium)}

\subsection{Bisphosphonates}

The structure of bisphosphonates resembles endogenous pyrophosphates that are able to bind to mineralized bone matrix in areas of high bone turnover. Bisphosphonates are able to inhibit tumor formation at the bone matrix, osteoclast development from precursor cells, and inhibit angiogenesis. Bisphosphonates can also initiate apoptosis of both osteoclasts and tumor cells.

Initial studies with a low-potency bisphosphonate, pamidronate, did not reduce SREs in prostate cancer patients [17]. However, a more potent bisphosphonate, zoledronic acid, reduced the incidence of SREs in a placebo-controlled randomized clinical trial of 122 men with metastatic castration-resistant prostate cancer. The annual incidence of SREs for men receiving zoledronic acid was 0.77 versus 1.47 for those receiving placebo $(P=0.005)$ while the median time to the first SRE was 488 days for men receiving zoledronic acid versus 321 days for those receiving placebo $(P=0.009)$ [18].

In breast cancer, the use of zoledronic acid in metastatic disease being treated with hormone therapy has demonstrated decreased incidence of SREs (as compared with treatment with pamidronate), in fact with an improved risk ratio compared with those receiving chemotherapy rather than hormones [19]. Given the effects on preventing and delaying SREs in men with castrate-resistant prostate cancer (CRPC), there has been interest in determining whether moving zoledronic acid into earlier phases of disease may prove effective. CALBG 90202 randomized men with castration-sensitive prostate cancer and bone metastases who were receiving androgen deprivation therapy to either zoledronic acid or placebo, with crossover to zoledronic acid for those who then developed CRPC. In this study, early treatment with zoledronic acid did not demonstrate an improvement in SREs compared with waiting until development of CRPC [20]. It is unclear why these two diseases may differ with respect to response to timing of bisphosphonate therapy, although it may be that androgen deprivation therapy is sufficiently active in castration-sensitive prostate cancer to overshadow any additional activity of bisphosphonates. 
Further upstream in the clinical phases of prostate cancer, there has been interest in altering the "soil" of the bone microenvironment with bisphosphonates to make it less hospitable to tumor cells with the goal of preventing the formation of metastatic disease. In the ZEUS trial, men with high-risk localized prostate cancer were randomized to receive zoledronic acid or placebo. With follow-up of almost 5 years, there was no difference in rates of bone metastases between the two groups [21]. In combination with the CALBG 90202 data, this trial provides supportive evidence for the practice of reserving bisphosphonate use until bone metastatic and castration-resistant disease develops.

\subsection{Denosumab}

Denosumab, a fully human monoclonal RANKL antibody, inhibits osteoclast maturation and bone turnover by mimicking the native osteoprotegerin-RANK interaction $[14,15]$. Denosumab has demonstrated activity both in the treatment of osteoporosis and the reduction of SREs in metastatic CRPC [22].

In a phase 2 clinical trial of denosumab versus zoledronic acid in patients with metastatic prostate cancer, breast cancer, multiple myeloma and other solid tumor patients, the denosumab arm had fewer SREs ( $8 \%$ vs. $17 \%$ ) as compared to ZA. The primary endpoint of urinary N-telopeptide (uNTx) lower than $50 \mathrm{nmol} / \mathrm{L} \mathrm{BCE} / \mathrm{mM}$ creatinine (uNTx<50) at week 13 was achieved by 49 (71\%) of 69 patients in the denosumab arms, compared with $10(29 \%)$ of 35 patients in the IV zoledronic acid arm $(P<0.001)$ [23].

These data prompted a large phase III trial to evaluate the role of denosumab in advanced prostate cancer. Men with CRPC were randomized to either zoledronic acid or denosumab with primary endpoint of first SRE, powered for non-inferiority, with superiority as a secondary endpoint. Compared with zoledronic acid, denosumab delayed the median time to first SRE by an additional 3.6 months $(H R=0.82$, non-inferiority $P=0.0002$, superiority $P=0.008)$. Hypocalcemia occurred in $13 \%$ of men in the denosumab group and in $1 \%$ of men in the zoledronic acid $(P>0.001)$ while osteonecrosis of the jaw occurred in $2 \%$ of men in the denosumab group and in $1 \%$ of men in the zoledronic acid group [24]. Based on these studies, the FDA approved the use of denosumab for the prevention of SREs in patients with solid tumors and as treatment to increase the bone mass in patients at high risk for fracture, including prostate cancer patients on hormonal treatment [22].

Denosumab's efficacy in extending bone metastasis-free survival (BMFS) was evaluated in another phase 3, double-blind, randomized, placebo-controlled study. One thousand four hundred thirty-two men with non-metastatic castration-resistant prostate cancer at high risk of bone metastasis prostate-specific antigen (PSA) $(\geq 8.0 \mu \mathrm{g} / \mathrm{L})$, PSA doubling time $(\leq 10.0$ months), or both were randomly assigned to receive subcutaneous denosumab $120 \mathrm{mg}$ or placebo every 4 weeks. Denosumab increased bone metastasis-free survival by a median of 4.2 months compared with placebo (29.5 vs. 25.2 months; $P=0.028)$ and delayed time to first bone metastasis by a median of 3.7 months (33.2 vs. 29.5 months; $P=0.032)$. Stratification by PSA doubling time (PSADT) revealed that patients with the shortest PSADT ( $\$ 4$ months) received the greatest benefit in terms of BMFS. Nevertheless, there was no statistically significant difference in overall survival (OS) between the placebo and treatment groups [25]. Post hoc exploratory analyses of time to symptomatic bone metastases as well as 
measures of pain and quality of life were either incomplete or did not demonstrate improvement. Thus, the FDA declined to approve denosumab for nonmetastatic CRPC, citing that a larger effect size for BMFS ( 1 year) would be needed in the setting of a lack of improvement of other clinical outcomes, that there was a potential for more extended use with subsequent potentially increased risks for complications such as hypocalcemia and osteonecrosis of the jaw [26].

Clinical decision-making regarding the use of zoledronic acid versus denosumab to reduce/ prevent SREs is not straightforward. Considerations include the long history of use and experience with zoledronic acid, whereas denosumab has a shorter track record. Zoledronic acid deposits in the bone have persistent effects whereas denosumab, as a monoclonal antibody, has a shorter duration of activity after administration. Denosumab has shown superiority over zoledronic acid with regards to prevention of SREs, but remains markedly more expensive than generic zoledronic acid with a statistically nonsignificant increase in osteonecrosis of the jaw and a greater risk of hypocalcemia. There may be benefits of ease of administration for denosumab, as zoledronic acid requires an intravenous infusion while denosumab is given subcutaneously. An additional benefit of denosumab is that it does not require evaluation of renal function with each dosing, although calcium levels do require monitoring.

\subsection{Radiopharmaceuticals}

One approach to target the bone microenvironment in prostate cancer is the use of radiopharmaceutical agents. Due to their similarity to calcium, these compounds are taken up at sites of osteoblastic activity and destroy the surrounding tissue through radiation emission. The first radiopharmaceuticals approved for pain relief in patients with metastatic prostate cancer to the bones were strontium-89 [27, 28] and samarium-153 [29, 30]. Neither of those agents has yet been shown to prolong survival in a large randomized study. Clinical use has thus been sparse and has been limited largely to patients with multiple simultaneously painful sites. Of note, one study in men with CRPC and bone metastases randomized patients receiving docetaxel to strontium-89, zoledronic acid, neither, or both. In this study, primary outcomes were clinical progression-free survival (CPFS; pain progression, SRE, or death) and cost-effectiveness. Secondary outcomes were time to SRE, total SREs, and overall survival. Strontium- 89 improved CPFS but not OS. Hematologic adverse effects, particularly leukopenia and thrombocytopenia, were frequent [31].

The advent of radium-223 is likely to further limit the use of strontium- 89 and samarium-153. Unlike strontium-89, which emits beta particles and samarium-153, which emits beta and gamma particles, radium-223, emits alpha particles, which travel shorter distances and deliver higher energy than beta or gamma particles. Radium-223 binds hydroxyapatite at sites of increased osteoblastic activity and was thus hypothesized to deliver high radiation doses to regions surrounding the tumor while decreasing toxicity to normal bone marrow. In a randomized, multicenter, placebo-controlled phase II study, 64 men with CRPC and bone pain who had previously received external beam radiotherapy at the most painful site were assigned to either four radium-223 injections or placebo given every 4 weeks. Primary endpoints were time to SREs and change in bone-alkaline 
phosphatase (ALP) concentration while secondary endpoints included toxic effects, time to PSA progression, and overall survival. Median relative change in bone-ALP during treatment was -65.6 vs. $9.3 \%$ in the radium-223 group and the placebo groups, respectively $(P<0.0001)$. Median time to PSA progression was 26 versus 8 weeks $(P=0.048)$ while the median overall survival was 65.3 vs. 46.4 weeks $(\mathrm{P}=0.066)$ in the radium-223 group and the placebo groups, respectively [32].

The phase III ALSYMPCA trial demonstrated an overall survival benefit for treatment with radium-223 in men with metastatic castration-resistant prostate cancer (mCRPC) patient who were either postdocetaxel or unfit for docetaxel, had multiple painful bone metastases, and no visceral disease. Median OS in the treatment arm was 14.0 months compared with 11.2 months in the placebo arm $(P=0.002)$ and there was also improvement in time to first SRE for the radium-223 group (13.6 vs. 8.4 months; $P<0.001)$. The trial met its end points in an early interim analysis [4]. Adverse events were minimal with no difference seen between the two arms and actually lower overall numbers of adverse events and serious adverse events in the treatment arm. Hematologic toxicity was relatively modest with similar rates of anemia compared with placebo and slightly increased rates of grade 3 ( 3 vs. $2 \%$ ) and grade 4 thrombocytopenia ( 3 vs. $<1 \%$ ) and grade $3(2$ vs. $1 \%)$ and grade $4(1$ vs. $0 \%)$ neutropenia. Radium-223 was FDA approved with an indication for men with symptomatic mCRPC with bone but no visceral metastases.

Radium-223 is the first therapy directed against the bone microenvironment and the first form of radiation therapy to show overall survival benefit in men with CRPC. As it appears to be well-tolerated, it may be considered prior to docetaxel-based chemotherapy. However, there are several challenges to its use. Time to pain relief after administration remains an important unknown given the frequent need for rapid palliation, although with one trial demonstrating a pain response rate of $71 \%$ at 2 weeks after administration, it appears promising in this regard [33]. Questions remain about the optimal dose and current use of flat dosing for all men despite varying burdens of metastatic disease. Additionally, the current FDA label restricts the use of radium-223 to bone-only disease-a distinction not present in the label for the first-generation radiopharmaceuticals. Lastly, given the logistical challenges of administering radiopharmaceuticals, clinical uptake will depend on fostering relationships between medical oncology and radiation oncology or nuclear medicine.

The relatively benign toxicity profile of radium-223 potentially lends itself to combination therapies with other active agents. In a phase I study of radium-223 and docetaxel, the combination appeared to be well-tolerated as compared to single agents with no delay of radium-223 due to adverse events and no reported long-term toxicity [34]. A phase II study is currently ongoing in an effort to explore the side effects and efficacy of the combination of radium-223 and docetaxel versus docetaxel alone (NCT01106352). The possible synergy between radium-223 and immunotherapy (sipuleucel-T) is also intriguing as radium-223 may facilitate release of antigens. Lastly, radium-223 may have the potential to combine well with potent hormonal therapies such as abiraterone and enzalutamide. Further trials are needed to explore these combinations as well as to determine the optimal timing and sequencing of radium-223 with other active agents. 


\subsection{Signal transduction pathway inhibition}

Inhibition of many of the signal transduction pathways thought to facilitate metastatic tumor growth within the bone microenvironment has unfortunately proved disappointing to date. However, simultaneous inhibition of multiple pathways, as demonstrated by cabozantinib, appears promising.

\subsection{ET1 receptor inhibitors}

ET-1 is a potent vasoconstrictor peptide hypothesized to play an important role in the progression of bone metastasis through its mitogenic activity in osteoblasts. Atrasentan and zibotentan, two inhibitors of the endothelin-A receptor, have demonstrated early evidence of activity in prostate cancer. In a phase II, double-blind, randomized, placebo-controlled clinical trial, 288 patients with mCRPC were randomly assigned to one of three study groups receiving either placebo, $2.5 \mathrm{mg}$ atrasentan, or $10 \mathrm{mg}$ atrasentan. In the fully evaluable subset of patients, $10 \mathrm{mg}$ atrasentan delayed time-to-progression ( $155 \mathrm{vs.} 71$ days; $P=0.002$ ) in comparison with placebo [35]. Similar data were presented for zibotentan [36]. However, phase III studies of atrasentan or zibotentan as single agents failed to show an increase in PFS or OS [37-40].

Both atrasentan and zibotentan were evaluated in combination with docetaxel. These phase III studies hypothesized that, given preclinical models demonstrating the potential for synergy [41], the combination might prove more effective. Both studies were negative. Given the consistency of negative data on clinical endpoints, the development of ET-1 inhibitors in prostate cancer has ceased.

\subsection{SRC inhibitors}

The nonreceptor tyrosine kinase SRC plays a significant role in tumor development within the bone microenvironment, regulating osteoclast function and metastasis physiology [4244]. SRC inhibitors, including dasatinib, saracatinib, and bosutinib, have shown activity in preclinical studies of prostate cancer, demonstrating inhibition of prostate cancer cell adhesion and invasion as well as tumor growth and skeletal lesions [45-48]. In a phase II clinical trial of saracatinib monotherapy in 28 men with CRPC, five men demonstrated a transient PSA reduction. No markers of skeletal-related events were evaluated in this study [49]. In a phase II trial in patients with chemotherapy-naive CRPC and increasing PSA levels ( $n=48)$, single-agent dasatinib had modest antitumor activity; after 12 and 24 weeks of therapy, $21(44 \%)$ and $8(17 \%)$ patients remained free from progressive disease. Use of dasatinib lead to decreased urinary $\mathrm{N}$-telopeptide levels (51\% of patients) and bone alkaline-phosphatase levels (59\% of patients).

Subsequently, a small phase II trial combined dasatinib with docetaxel, demonstrating bone scan improvements in $30 \%$ of patients with 87 and $76 \%$ with improvements in urinary Ntelopeptide and bone-specific ALP, respectively [50]. On the basis of these data, the phase III READY trial randomized 1,522 patients with $\mathrm{mCRPC}$ to receive either docetaxel 75 $\mathrm{mg} / \mathrm{m}^{2}$ every 3 weeks plus prednisone with dasatinib $100 \mathrm{mg}$ every day or docetaxel plus placebo. There was no statistically significant difference in the median OS between the dasatinib/docetaxel group versus the docetaxel/ placebo group (21.5 vs. 21.2 months, 
respectively) while there were no meaningful changes between the two arms for secondary endpoints, including objective response rate, reduction in urinary $\mathrm{N}$-telopeptide, progression-free survival, or pain reduction. However, there was a suggestion of delayed time to first SRE in the dasatinib arm with the median time not yet reach versus 31.1 months in the placebo arm. This finding must be considered exploratory given the negative primary endpoint. Thus, despite the preclinical evidence that SRC signaling plays a key role in osteoclast function, late-phase trials have dampened enthusiasm for continued study in prostate cancer.

\subsection{Thalidomide and lenalidomide}

Thalidomide and lenalidomide demonstrate a multitude of properties potentially beneficial for metastatic prostate cancer including antiangiogenic effects through reduction of VEGF and basic fibroblast growth factor. In addition, these agents suppress osteoclast differentiation, act to inhibit adhesion in the bone microenvironment, and limit bone marrow stromal cell support of tumor cells [51, 52]. Lastly, they act as immunomodulators via cytokines including interleukin (IL)-6, IL-10, and tumor necrosis factor alpha [53]. In a randomized phase II trial combining docetaxel with thalidomide versus docetaxel alone, the addition of thalidomide yielded a nonstatistically significant increase in median overall survival [54]. However, there were no responses by bone scan. While thalidomide was not tested in a phase III setting, a subsequent phase III trial of lenalidomide in combination with docetaxel and prednisone (MAINSAIL) failed to confirm any benefit and was terminated prematurely after the data monitoring committee deemed futility in achieving its primary endpoint of OS. Additionally, this study showed that combination therapy had greater toxicity and significantly more deaths ( $>28$ days from last lenalidomide dose) than the standard therapy arm (20.8 vs. $15 \% ; P=0.016)$ [55].

\subsection{Cabozantinib}

Cabozantinib (XL184), is a multikinase inhibitor that primarily targets the mesenchymal epithelial transition factor and the vascular endothelial growth factor receptor 2, and has activity against other receptor tyrosine kinases (FLT3, RET, KIT, and TIE2) [56]. In preclinical studies, the MET and VEGF pathways have been postulated to interact to promote angiogenesis and the MET pathway has effects on osteoblasts through BMP-2 [57, 58]. These multitargeted features potentially distinguish cabozantinib from earlier agents targeting these receptors individually, such as bevacizumab for VEGF and rilotumumab for MET, which have not demonstrated significant clinical benefits [59, 60]. What have excited the prostate cancer community are the dramatic improvements in bone scans, with $68 \%$ of men decreasing uptake after treatment with complete resolution in $12 \%$. Importantly, these responses were associated with decreases in bone pain [61]. However, there remains concern that the responses on bone scans may represent decreased metabolic activity rather than true response, almost like a "cloaking mechanism." Further radiographic studies evaluating the effect on bone lesions using CT and MRI are awaited. Moreover, while generally fairly well-tolerated at the doses used ( $100 \mathrm{mg} /$ day), adverse events including fatigue, hypertension, and hand-foot syndrome have been a concern and $51 \%$ of the patients required dose reductions. 
There is currently an ongoing phase III trial (COMET-1; ClinicalTrials.gov identifier: NCT01605227) of cabozantinib using a lower dose (60 mg/day) versus prednisone in patients with mCRPC previously treated with docetaxel and abiraterone or enzalutamide with a primary endpoint of OS. Another ongoing phase III trial (COMET-2;

ClinicalTrials.gov identifier: NCT01522443) compares the effect cabozantanib on pain and bone scan response in men with CRPC and painful bone metastases with a comparator arm of mitoxantrone plus prednisone.

\subsection{Tasquinimod}

Tasquinimod, derived from quinoline- 3 carboxamide [62, 63], has been proposed to have immunomodulatory properties through S100-9, a protein involved in differentiation and cell cycle as well as antisurvival and anti-angiogenic properties through inhibition of HDAC4, leading to downregulation of hypoxia inducible factor [64, 65]. In an intratibial xenograft model, tasquinimod inhibited establishment of CRPC tumors in the bone [66]. During subsequent phase I evaluation in men with CRPC, $80 \%$ of patients with bone metastases at the start of trial had no evidence of progression after a median of 34 weeks of treatment [67]. In a phase II randomized, double-blind, placebo-controlled study of tasquinimod, 221 men with minimally symptomatic metastatic castration-resistant prostate cancer were assigned (2:1) to receive either oral once-daily tasquinimod $0.25 \mathrm{mg}$ /day escalating to 1.0 $\mathrm{mg} /$ day over 4 weeks or placebo. The 6-month PFS for tasquinimod and placebo groups were 69 and $37 \%$, respectively $(P<0.001)$, while the median PFS was 7.6 versus 3.3 months $(P=0.0042)$ with a trend towards improved OS. Adverse events (AEs) occurring more frequently with tasquinimod included gastrointestinal disorders, fatigue, musculoskeletal pains, and elevations of pancreatic and inflammatory biomarkers. Grades 3 to 4 AEs, including asymptomatic elevations of laboratory parameters and deep vein thrombosis were also reported [68, 69]. Currently, a phase III study of tasquinimod in chemotherapy-naïve patients with mCRPC with a primary endpoint of radiographic PFS is maturing (ClinicalTrials.gov identifier: NCT01234311).

\section{Novel androgen-directed agents}

Androgen receptor (AR) signaling has a key role in the progression of prostate cancer. Demonstrating the premise that effective therapy for prostate cancer yields effective therapy for bone metastases, two novel agents, abiraterone acetate and enazalutamide, have demonstrated improvement in bone-related endpoints as well as overall survival, despite not being designed to specifically target the bone. These agents have been subsequently approved by the FDA after demonstrating overall survival benefits after chemotherapy (abiraterone and enzalutamide) and before chemotherapy (abiraterone) [5, 70, 71].

Abiraterone acetate is a selective androgen biosynthesis inhibitor that blocks CYP17, disrupting androgen production in the adrenals, testes, and prostate cancer cells. The COUAA-301 study randomized docetaxel-experienced men with mCRPC to either abiraterone/prednisone or placebo/ prednisone. In addition to OS benefits, patients in the abiraterone arm demonstrated significantly longer radiographic PFS compared to placebo (5.6 vs. 3.6 months; $P<0.0001)$ as well as increased time to first SRE (25.0 vs. 20.3 months; $P=0.0001$ ) and improved and more rapid palliation of pain (5.6 vs. 13.7 months; $P=0.0018$ ) 
[5]. These findings are all the more impressive given that approximately half the patients in each arm received bisphosphonate treatment during the course of the study, indicating boneprotective activity additive to that of bisphosphonates alone [72]. In the chemotherapy-naïve COU-AA-302 study, time to first SRE was not a prespecified secondary endpoint; however, there were statistically significant improvements in median time to opiate use (NR vs. 23.7 months; $P<0.001)$ and increase in pain outcomes (26.7 vs. 18.4 months; $P=0.049)$ [71].

Enzalutamide is an AR antagonist that targets multiple sites of the androgen pathway and has approximately fivefold higher binding affinity for the AR compared to the anti-androgen bicalutamide [73]. In the phase III AFFIRM trial, men with mCRPC who had progressed after chemotherapy were randomized to enzalutamide or placebo. Overall survival was significantly improved in the enzalutamide arm (18.4 vs. 13.6 months; $P<0.001)$ [70]. In a sub-analysis focusing on bone-related measures, enzalutamide demonstrated a significant delay in time to first SRE in comparison with the placebo group (16.7 vs. 13.3 months, respectively; $P=0.0001$ ) leading to a $31 \%$ reduction in risk and increased the radiographic PFS in comparison with the placebo group (8.3 vs. 2.9 months; $P<0.001)$ [74].

It is unsurprising that treatments that are globally active in prostate cancer are similarly active in bone metastatic disease; however, it is notable that the effect of inhibiting the androgen receptor carries incremental benefit in preventing SREs and treating bone pain over that of bisphosphonates alone. It will be interesting to note whether these benefits persist in the era of denosumab.

\section{Future directions}

Given the success of zoledronic acid, denosumab, and the several recent survival-enhancing drugs and perhaps due to the failure of other inhibitors of apparently important pathways in the bone microenvironment, there have been several promising agents that have not yet moved forward into large trials. For example, odanacatib, an inhibitor of cathepsin K, a cysteine protease expressed by osteoclasts which cleaves collagen I and promotes osteoclast adherence to the extracellular matrix, has shown efficacy in preventing SREs in breast cancer [75]. However, no trials in CRPC are currently ongoing.

Targeting of hematopoetic stem cells (HSCs) in the bone marrow microenvironment may also prove effective at preventing or treating metastatic disease. Within the microenvironment, HSCs are able to enter a dormant state to preserve self-renewal capacity, while retaining the ability to divide and differentiate when there is need [76]. DTCs preferentially target this niche [77] where they either remain dormant or proliferate and eventually metastasize [78]. In a recent pre-clinical study in which DTCs were introduced into immuno-deficient mice and allowed to populate the bone marrow, DTCs were able to target and displace HSCs out of their niche and establish a metastatic locus [10]. This targeting appears to at least in part be mediated by osteoblast secretion of high quantities of the chemokine SDF01 (CXCL12), attracting prostate cancer cells which express the chemokine receptors CXCR4 and CXCR7, as do HSCs. Inhibition of the SDF-1/ CXCR4 axis, through the use of agents such as AMD3100 (plerixafor) that are able to mobilize HSCs, could potentially disrupt the connection between disseminating prostate cancer cells 
and the onco-niche. Prostate cancer cells mobilized from their protective onco-niche to the peripheral blood and forced to exit their dormant state may also be more vulnerable to chemotherapy.

\section{Conclusions}

The hallmark of advanced prostate cancer is metastasis to the bone. While therapies affecting the osteoclast/osteoblast equilibrium have shown efficacy with regards to preventing SREs and, in the case of denosumab, increase bone metastasis-free survival, they have not demonstrated the ability to increase overall survival. Many drugs targeting pathways involved in the bone marrow microenvironment have been disappointing despite promising preclinical data, including ET-1, SRC, and VEGF, although the multitargeted agent, cabozantinib, appears promising. The success of radium-223 has opened new frontiers in the management of advanced prostate cancer, specifically focused on the bone microenvironment, and has the potential to be combined with other active therapies. Potent drugs targeting the androgen receptor also provide benefits in bone metastases as demonstrated by recent data from abiraterone and enzalutamide. As the pathways governing osteoblast-osteoclast equilibrium and the bone microenvironment are better understood, more targeted treatments can be identified with the goal of transforming lethal prostate cancer into a chronic disease.

\section{References}

1. Tannock IF, de Wit R, Berry WR, et al. Docetaxel plus prednisone or mitoxantrone plus prednisone for advanced prostate cancer. The New England Journal of Medicine. 2004; 351(15):1502-1512. [PubMed: 15470213]

2. Kantoff PW, Higano CS, Shore ND, et al. Sipuleucel-T immunotherapy for castration-resistant prostate cancer. The New England Journal of Medicine. 2010; 363(5):411-422. [PubMed: 20818862]

3. de Bono JS, Oudard S, Ozguroglu M, et al. Prednisone plus cabazitaxel or mitoxantrone for metastatic castration-resistant prostate cancer progressing after docetaxel treatment: a randomised open-label trial. The Lancet. 2010; 376(9747):1147-1154.

4. Parker C, Nilsson S, Heinrich D, et al. Alpha emitter radium-223 and survival in metastatic prostate cancer. The New England Journal of Medicine. 2013; 369(3):213-223. [PubMed: 23863050]

5. Fizazi K, Scher HI, Molina A, et al. Abiraterone acetate for treatment of metastatic castrationresistant prostate cancer: final overall survival analysis of the COU-AA-301 randomised, doubleblind, placebo-controlled phase 3 study. The Lancet Oncology. 2012; 13:983-992. [PubMed: 22995653]

6. Scher HI, Fizazi K, Saad F, et al. Effect of MDV3100, an androgen receptor signaling inhibitor (ARSI), on overall survival in patients with prostate cancer postdocetaxel: results from the phase III AFFIRM study. Journal of Clinical Oncology. 2012; 30(Suppl 5):1. [PubMed: 22105825]

7. Paget $\mathrm{S}$. The distribution of secondary growths in cancer of the breast. The Lancet. 1889; 133(3421):571-573.

8. Suda T, Takahashi N, Udagawa N, Jimi E, Gillespie MT, Martin TJ. Modulation of osteoclast differentiation and function by the new members of the tumor necrosis factor receptor and ligand families. Endocrine Reviews. 1999; 20(3):345-357. [PubMed: 10368775]

9. Wada T, Nakashima T, Hiroshi N, Penninger JM. RANKL-RANK signaling in osteoclastogenesis and bone disease. Trends in Molecular Medicine. 2006; 12(1):17-25. [PubMed: 16356770] 
10. Shiozawa Y, Pedersen EA, Havens AM, et al. Human prostate cancer metastases target the hematopoietic stem cell niche to establish footholds in mouse bone marrow. The Journal of Clinical Investigation. 2011; 121(4):1298. [PubMed: 21436587]

11. Pedersen EA, Shiozawa Y, Pienta KJ, Taichman RS. The prostate cancer bone marrow niche: more than just 'fertile soil'. Asian Journal of Andrology. 2012; 14(3):423-427. [PubMed: 22367179]

12. Schneider A, Kalikin LM, Mattos AC, et al. Bone turnover mediates preferential localization of prostate cancer in the skeleton. Endocrinology. 2005; 146(4):1727-1736. [PubMed: 15637291]

13. Shiozawa Y, Pedersen EA, Patel LR, et al. GAS6/AXL axis regulates prostate cancer invasion, proliferation, and survival in the bone marrow niche. Neoplasia (New York, NY). 2010; 12(2):116.

14. Taichman RS, Patel LR, Bedenis R, et al. GAS6 receptor status is associated with dormancy and bone metastatic tumor formation. PloS one. 2013; 8(4):e61873. [PubMed: 23637920]

15. Loberg RD, Gayed BA, Olson KB, Pienta KJ. A paradigm for the treatment of prostate cancer bone metastases based on an understanding of tumor cell-microenvironment interactions. Journal of Cellular Biochemistry. 2005; 96(3):439-446. [PubMed: 15988761]

16. Guise TA, Mohammad KS, Clines G, et al. Basic mechanisms responsible for osteolytic and osteoblastic bone metastases. Clinical cancer research. 2006; 12(20):6213s-6216s. [PubMed: 17062703]

17. Small EJ, Smith MR, Seaman JJ, Petrone S, Kowalski MO. Combined analysis of two multicenter, randomized, placebo-controlled studies of pamidronate disodium for the palliation of bone pain in men with metastatic prostate cancer. Journal of Clinical Oncology. 2003; 21(23):4277-4284. [PubMed: 14581438]

18. Saad F, Gleason DM, Murray R, et al. Long-term efficacy of zoledronic acid for the prevention of skeletal complications in patients with metastatic hormone-refractory prostate cancer. Journal of the National Cancer Institute. 2004; 96(11):879-882. [PubMed: 15173273]

19. Rosen LS, Gordon D, Kaminski M, et al. Long-term efficacy and safety of zoledronic acid compared with pamidronate disodium in the treatment of skeletal complications in patients with advanced multiple myeloma or breast carcinoma. Cancer. 2003; 98(8):1735-1744. [PubMed: 14534891]

20. Smith MR, Halabi S, Ryan CJ, et al. Efficacy and safety of zoledronic acid in men with castrationsensitive prostate cancer and bone metastases: results of CALGB 90202 (alliance). ASCO Meeting Abstracts. 2013; 31(6_suppl):27.

21. Wirth M, Tammela T, Huland H, et al. Zoledronic acid for the prevention of bone metastases in high risk prostate cancer patients. A randomised, open label, multicentre study of the European Association of Urology (EAU) in cooperation with the Scandinavian Prostate Cancer Group (SPCG) and the Arbeitsgemeinschaft Urologische Onkologie (AUO). European Urology Supplements. 2008; 7(3):232.

22. Smith MR, Egerdie B, Toriz NH, et al. Denosumab in men receiving androgen-deprivation therapy for prostate cancer. The New England Journal of Medicine. 2009; 361(8):745-755. [PubMed: 19671656]

23. Fizazi K, Lipton A, Mariette X, et al. Randomized phase II trial of denosumab in patients with bone metastases from prostate cancer, breast cancer, or other neoplasms after intravenous bisphosphonates. Journal of Clinical Oncology. 2009; 27(10):1564-1571. [PubMed: 19237632]

24. Fizazi K, Carducci M, Smith M, et al. Denosumab versus zoledronic acid for treatment of bone metastases in men with castration-resistant prostate cancer: a randomised, double-blind study. The Lancet. 2011; 377(9768):813-822.

25. Smith MR, Saad F, Coleman R, et al. Denosumab and bone-metastasis-free survival in men with castration-resistant prostate cancer: results of a phase 3, randomised, placebo-controlled trial. The Lancet. 2012; 379(9810):39-46.

26. Summary minutes of the Oncologic Drugs Advisory Committee meeting. 2012

27. Silberstein EB. Dosage and response in radiopharmaceutical therapy of painful osseous metastases. Journal of Nuclear Medicine. 1996; 37(2):249-252. [PubMed: 8667054]

28. Tu S, Millikan RE, Mengistu B, et al. Bone-targeted therapy for advanced androgen-independent carcinoma of the prostate: a randomised phase II trial. The Lancet. 2001; 357(9253):336-341. 
29. Sartor O, Reid RH, Bushnell DL, Quick DP, Ell PJ. Safety and efficacy of repeat administration of samarium Sm-153 lexidronam to patients with metastatic bone pain. Cancer. 2007; 109(3):637643. [PubMed: 17167764]

30. Fizazi K, Beuzeboc P, Lumbroso J, et al. Phase II trial of consolidation docetaxel and samarium-153 in patients with bone metastases from castration-resistant prostate cancer. Journal of Clinical Oncology. 2009; 27(15):2429-2435. [PubMed: 19364971]

31. James ND, Pirrie S, Barton D, et al. Clinical outcomes in patients with castrate-refractory prostate cancer (CRPC) metastatic to bone randomized in the factorial TRAPEZE trial to docetaxel (D) with strontium-89 (Sr89), zoledronic acid (ZA), neither, or both (ISRCTN 12808747). ASCO Meeting Abstracts. 2013; 31(18_suppl):LBA5000.

32. Nilsson S, Franzén L, Parker C, et al. Bone-targeted radium-223 in symptomatic, hormonerefractory prostate cancer: a randomised, multicentre, placebo-controlled phase II study. The Lancet Oncology. 2007; 8(7):587-594. [PubMed: 17544845]

33. Nilsson S, Strang P, Aksnes AK, et al. A randomized, dose-response, multicenter phase II study of radium-223 chloride for the palliation of painful bone metastases in patients with castrationresistant prostate cancer. European Journal of Cancer. 2012; 48(5):678-686. [PubMed: 22341993]

34. Morris MJ, Hammers HJ, Sweeney C, et al. Safety of radium-223 dichloride (ra-223) with docetaxel (D) in patients with bone metastases from castration-resistant prostate cancer (CRPC): a phase I prostate cancer clinical trials consortium study. ASCO Meeting Abstracts. 2013; 31(15_suppl):5021.

35. Carducci MA, Padley RJ, Breul J, et al. Effect of endothelin-A receptor blockade with atrasentan on tumor progression in men with hormone-refractory prostate cancer: a randomized, phase II, placebo-controlled trial. Journal of Clinical Oncology. 2003; 21(4):679-689. [PubMed: 12586806]

36. Schelman WR, Liu G, Wilding G, Morris T, Phung D, Dreicer R. A phase I study of zibotentan (ZD4054) in patients with metastatic, castrate-resistant prostate cancer. Investigational New Drugs. 2011; 29(1):118-125. [PubMed: 19763400]

37. James ND, Caty A, Payne H, et al. Final safety and efficacy analysis of the specific endothelin A receptor antagonist zibotentan (ZD4054) in patients with metastatic castration-resistant prostate cancer and bone metastases who were pain-free or mildly symptomatic for pain: a double-blind, placebo-controlled, randomized phase II trial. BJU International. 2010; 106(7):966-973. [PubMed: 20840318]

38. Nelson JB, Love W, Chin JL, et al. Phase 3, randomized, controlled trial of atrasentan in patients with nonmetastatic, hormone-refractory prostate cancer. Cancer. 2008; 113(9):2478-2487. [PubMed: 18785254]

39. Nelson JB, Fizazi K, Miller K, et al. Phase 3, randomized, placebo-controlled study of zibotentan (ZD4054) in patients with castration-resistant prostate cancer metastatic to bone. Cancer. 2012; 118(22):5709-5718. [PubMed: 22786751]

40. Miller K, Moul J, Gleave M, et al. Phase III, randomized, placebo-controlled study of once-daily oral zibotentan (ZD4054) in patients with non-metastatic castration-resistant prostate cancer. Prostate cancer and prostatic diseases. 2013; 16(2):187-192. [PubMed: 23381694]

41. Akhavan A, McHugh KH, Guruli G, et al. Endothelin receptor A blockade enhances taxane effects in prostate cancer. Neoplasia. 2006; 8(9):725-732. [PubMed: 16984730]

42. Kim LC, Song L, Haura EB. Src kinases as therapeutic targets for cancer. Nature Reviews Clinical Oncology. 2009; 6(10):587-595.

43. Summy JM, Gallick GE. Src family kinases in tumor progression and metastasis. Cancer and Metastasis Reviews. 2003; 22(4):337-358. [PubMed: 12884910]

44. Edwards J. Src kinase inhibitors: an emerging therapeutic treatment option for prostate cancer. Expert Opinion on Investigational Drugs. 2010; 19(5):605-614. [PubMed: 20367532]

45. Tatarov O, Mitchell TJ, Seywright M, Leung HY, Brunton VG, Edwards J. SRC family kinase activity is up-regulated in hormone-refractory prostate cancer. Clinical Cancer Research. 2009; 15(10):3540-3549. [PubMed: 19447874]

46. Nam S, Kim D, Cheng JQ, et al. Action of the src family kinase inhibitor, dasatinib (BMS-354825), on human prostate cancer cells. Cancer Research. 2005; 65(20):9185-9189. [PubMed: 16230377] 
47. Brownlow N, Mol C, Hayford C, Ghaem-Maghami S, Dibb N. Dasatinib is a potent inhibitor of tumour-associated macrophages, osteoclasts and the FMS receptor. Leukemia. 2008; 23(3):590 594. [PubMed: 18784745]

48. Lee Y, Huang C, Murshed M, Chu K, Araujo JC, Ye X. Src family kinase/abl inhibitor dasatinib suppresses proliferation and enhances differentiation of osteoblasts. Oncogene. 2010; 29(22): 3196-3207. [PubMed: 20228840]

49. Araujo JC, Trudel GC, Saad F, Armstrong AJ, Yu EY, Bellmunt J, et al. Docetaxel and dasatinib or placebo in men with metastatic castration-resistant prostate cancer (READY): a randomised, double-blind phase 3 trial. Lancet Oncol. 2013; 14(13):1307-1316. [PubMed: 24211163]

50. Araujo JC, Mathew P, Armstrong AJ, et al. Dasatinib combined with docetaxel for castrationresistant prostate cancer. Cancer. 2012; 118(1):63-71. [PubMed: 21976132]

51. Anderson G, Gries M, Kurihara N, et al. Thalidomide derivative CC-4047 inhibits osteoclast formation by down-regulation of PU.1. Blood. 2006; 107(8):3098-3105. [PubMed: 16373662]

52. Efstathiou E, Troncoso $\mathrm{P}$, Wen $\mathrm{S}$, et al. Initial modulation of the tumor microenvironment accounts for thalidomide activity in prostate cancer. Clinical Cancer Research. 2007; 13(4):1224-1231. [PubMed: 17317833]

53. Vallet S, Palumbo A, Raje N, et al. Thalidomide and lenalidomide: mechanism-based potential drug combinations. Leuk Lymphoma. 2008; 49(7):1238-1245. [PubMed: 18452080]

54. Dahut WL, Gulley JL, Arlen PM, et al. Randomized phase II trial of docetaxel plus thalidomide in androgen-independent prostate cancer. Journal of Clinical Oncology. 2004; 22(13):2532-2539. [PubMed: 15226321]

55. Petrylak D, Fizazi K, Sternberg C, Budnik N, Wit Rd WP. A phase 3 study to evaluate the efficacy and safety of docetaxel and prednisone (DP) with or without lenalidomide in patients with castrate-resistant prostate cancer (CRPC). the MAINSAIL trial. 2012

56. Chott A, Sun Z, Morganstern D, et al. Tyrosine kinases expressed in vivo by human prostate cancer bone marrow metastases and loss of the type 1 insulin-like growth factor receptor. The American Journal of Pathology. 1999; 155(4):1271-1279. [PubMed: 10514409]

57. Sulpice E, Ding S, Muscatelli-Groux B, et al. Cross-talk between the VEGF-A and HGF signalling pathways in endothelial cells. Biology of the Cell. 2009; 101(9):525-539. [PubMed: 19281453]

58. Tsai S, Huang Y, Yang W, Tang C. Hepatocyte growth factor-induced BMP-2 expression is mediated by c-met receptor, FAK, JNK, Runx2, and p300 pathways in human osteoblasts. International Immunopharmacology. 2012; 13(2):156-162. [PubMed: 22504529]

59. Ryan CJ, Rosenthal M, Ng S, et al. Targeted MET inhibition in castration-resistant prostate cancer: a randomized phase II study and biomarker analysis with rilotumumab plus mitoxantrone and prednisone. Clinical Cancer Research. 2013; 19(1):215-224. [PubMed: 23136195]

60. Kelly WK, Halabi S, Carducci M, et al. Randomized, double-blind, placebo-controlled phase III trial comparing docetaxel and prednisone with or without bevacizumab in men with metastatic castration-resistant prostate cancer: CALGB 90401. Journal of Clinical Oncology. 2012; 30(13): 1534-1540. [PubMed: 22454414]

61. Smith DC, Smith MR, Sweeney C, et al. Cabozantinib in patients with advanced prostate cancer: results of a phase II randomized discontinuation trial. Journal of Clinical Oncology. 2013; 31(4): 412-419. [PubMed: 23169517]

62. Isaacs JT, Pili R, Qian DZ, et al. Identification of ABR-215050 as lead second generation quinoline-3-carboxamide anti-angiogenic agent for the treatment of prostate cancer. Prostate. 2006; 66(16):1768-1778. [PubMed: 16955399]

63. Isaacs JT. The long and winding road for the development of tasquinimod as an oral secondgeneration quinoline-3-carboxamide antiangiogenic drug for the treatment of prostate cancer. Expert Opinion on Investigational Drugs. 2010; 19(10):1235-1243. [PubMed: 20836618]

64. Olsson A, Björk A, Vallon-Christersson J, Isaacs JT, Leanderson T. Tasquinimod (ABR-215050), a quinoline-3-carboxamide anti-angiogenic agent, modulates the expression of thrombospondin-1 in human prostate tumors. Molecular Cancer. 2010; 9(1):107. [PubMed: 20470445]

65. Isaacs JT, Antony L, Dalrymple SL, et al. Tasquinimod is an allosteric modulator of HDAC4 survival signaling within the compromised cancer microenvironment. Cancer Research. 2013; 73(4):1386-1399. [PubMed: 23149916] 
66. Jennbacken K, Welén K, Olsson A, et al. Inhibition of metastasis in a castration resistant prostate cancer model by the quinoline-3-carboxamide tasquinimod (ABR-215050). Prostate. 2012; 72(8): 913-924. [PubMed: 22287276]

67. Bratt O, Häggman M, Ahlgren G, Nordle Ö, Björk A, Damber J. Open-label, clinical phase I studies of tasquinimod in patients with castration-resistant prostate cancer. British Journal of Cancer. 2009; 101(8):1233-1240. [PubMed: 19755981]

68. Pili R, Häggman M, Stadler WM, et al. Phase II randomized, double-blind, placebo-controlled study of tasquinimod in men with minimally symptomatic metastatic castrate-resistant prostate cancer. Journal of Clinical Oncology. 2011; 29(30):4022-4028. [PubMed: 21931019]

69. Armstrong AJ, Haggman M, Stadler WM, et al. Tasquinimod and survival in men with metastatic castration-resistant prostate cancer: results of long-term follow-up of a randomized phase II placebo-controlled trial. Journal of Clinical Oncology. 2012; 30(15 Suppl):4550. [PubMed: 23169508]

70. Scher HI, Fizazi K, Saad F, et al. Increased survival with enzalutamide in prostate cancer after chemotherapy. The New England Journal of Medicine. 2012; 367(13):1187-1197. [PubMed: 22894553]

71. Ryan CJ, Smith MR, de Bono JS, et al. Abiraterone in metastatic prostate cancer without previous chemotherapy. The New England Journal of Medicine. 2013; 368(2):138-148. [PubMed: 23228172]

72. Logothetis CJ, Basch E, Molina A, et al. Effect of abiraterone acetate and prednisone compared with placebo and prednisone on pain control and skeletal-related events in patients with metastatic castration-resistant prostate cancer: exploratory analysis of data from the COU-AA-301 randomised trial. The lancet oncology. 2012; 13:1210-7. [PubMed: 23142059]

73. Tran C, Ouk S, Clegg NJ, et al. Development of a second-generation antiandrogen for treatment of advanced prostate cancer. Science. 2009; 324(5928):787-790. [PubMed: 19359544]

74. Fizazi K, Scher H, Saad F, et al. Impact of enzalutamide, an androgen receptor signaling inhibitor, on time to first skeletal related event (SRE) and pain in the phase 3 AFFIRM study. Annals of Oncology. 2012; 23:295-296.

75. Jensen AB, Wynne C, Ramirez G, et al. The cathepsin K inhibitor odanacatib suppresses bone resorption in women with breast cancer and established bone metastases: results of a 4-week, double-blind, randomized, controlled trial. Clinical Breast Cancer. 2010; 10(6):452-458. [PubMed: 21147688]

76. Taichman RS. Blood and bone: two tissues whose fates are intertwined to create the hematopoietic stem-cell niche. Blood. 2005; 105(7):2631-2639. [PubMed: 15585658]

77. Joyce JA, Pollard JW. Microenvironmental regulation of metastasis. Nature Reviews Cancer. 2008; 9(4):239-252.

78. Aguirre-Ghiso JA. Models, mechanisms and clinical evidence for cancer dormancy. Nature Reviews Cancer. 2007; 7(11):834-846. 
A

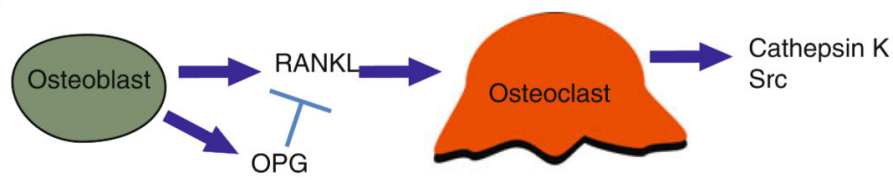

$\mathrm{B}$

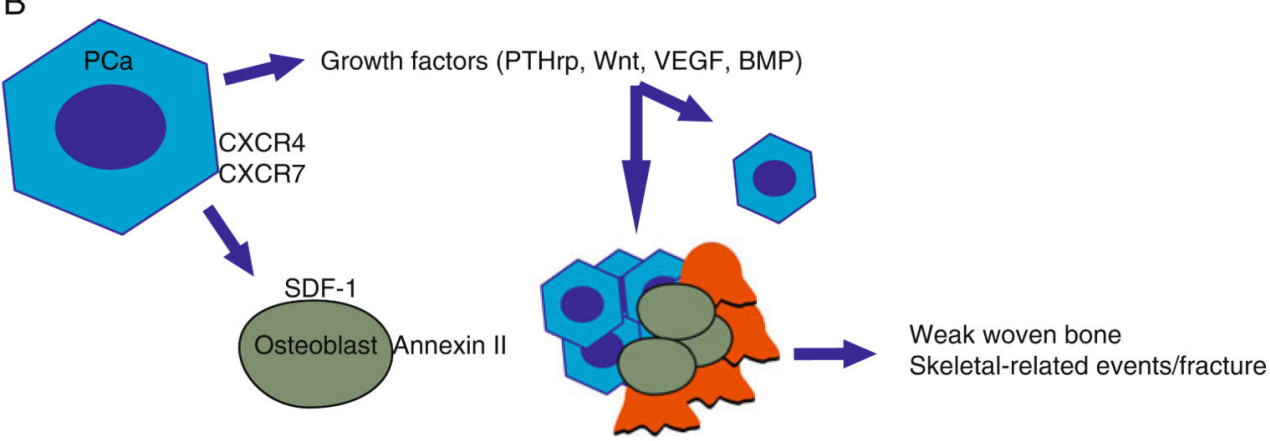

C

Atrasentan
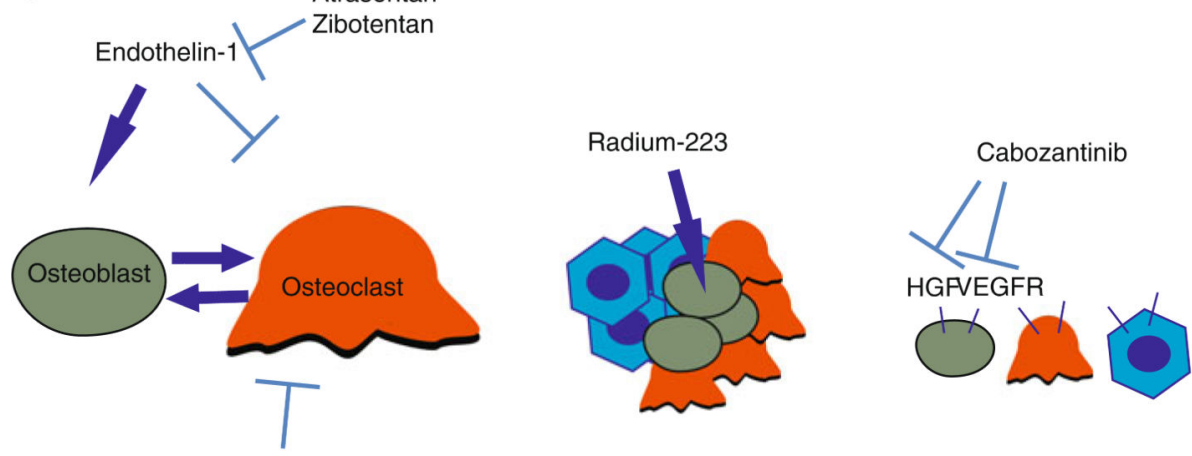

Bisphosphonates Denosumab

Fig. 1.

a In the normal bone microenvironment, osteoblast/osteoclast equilibrium is maintained via the RANK ligand (RANKL) secreted by osteoblasts as well as the decoy RANK receptor, osteoprotegerin $(O P G)$. RANKL stimulates osteoclasts to produce cathepsin $\mathrm{K}$, which catabolizes bone, as well as Src, which signals to promote osteoclast maturation and survival. b Hematopoetic stem cells home to bone via the SDF-1/CXCR4 axis. Prostate cancer disseminated tumor cells co-opt this mechanism through expression of the CXCR4 chemokine receptor, which homes to the SDF-1 chemokine secreted by osteoblasts. Once seeded in the bone, tumor cells secrete paracrine growth factors. Tumor cells branch either to dormancy or proliferation through a poorly understood mechanism. Proliferating tumor cells stimulate osteoclasts and osteoblasts, leading to weak woven bone and ultimately to skeletal-related events including pain and fracture. $\mathbf{c}$ Bisphosphonates coordinate calcium ions in bone via their phosphonate groups and inhibit osteoclast activity via the HMG-CoA reductase pathway. Denosumab directly blocks RANKL activity, preventing osteoclast proliferation. Endothelin, produced by benign prostatic epithelial cells, is mitogenic for prostate tumor cells and osteoblasts and is blocked by the ET-A receptor antagonists, atrasentan, and zibotentan. Radium-223 acts as a calcium mimetic and is taken up in hydroxyapatite where released alpha particles deliver short-distance high-energy radiation to 
surrounding tumor, osteoblasts, and osteoclasts. Cabozantinib blocks the HGF/MET and VEGF pathways present in prostate tumor cells, osteoblasts, and osteoclasts and additionally prevent interaction between the two pathways, which may be involved in angio-genesis, as well as proliferation, migration, and survival signals for all three cell types 